\title{
AMAZING BEAUTY AND HEALTH BENEFITS OF BANANAS
}

\section{*Prof. J .Gladys}

\section{Introduction}

Banana, plantain or Musa paradisiacal is a large, herbaceous plant native to India and Southeast Asia. It is one of the oldest cultivated plants. There are several types of bananas which are classified according to their taste and the color of their skin. Red bananas have a red peel and the flesh of the fruit is light pink in color. Red bananas are best if they are allowed to ripen fully at room temperature. Apple bananas are smaller than the usual varieties of bananas and are yellow in color. They tend to ripen much faster. The baby banana is one of the smallest varieties of bananas and they are yellow in color. They are very sweet and are perfect for infants and toddlers. All parts of the banana plant have medicinal applications. It has a rare combination of energy value, tissue-building elements, protein, vitamins and minerals. It is a good source of calories since it is rich in solids and low in water content as compared to any other fresh fruit.

\section{Provides instant energy:-}

Bananas contain three natural sugars sucrose, fructose and glucose combined with fiber. A banana gives an instant, sustained and substantial boost of energy. Research has proven that just two bananas provide enough energy for a strenuous 90-minute workout. Bananas have a healthier blend of sugars than sports drinks.

\section{* Maintains Blood pressure}

Bananas are a rich source of potassium, as well as calcium and magnesium Bananas are naturally fat and cholesterol free.Pectin, a fibre which helps to reduce cholesterol is found abundantly in bananas. Research has proven that natural compounds in bananas act in a manner similar to anti-hypertensive drugs. Also researches say that blood pressure fell by $10 \%$ in people who ate two bananas daily for a week. A team studied six popular banana varieties and found that all had ACEinhibiting properties, though the ripened bananas had a stronger action than unripe ones.

\section{* Maintains Blood Glucose Level}

One medium banana provides about 3 grams of fiber. Studies have shown that type 1 diabetics who consume high-fiber diets have lower blood glucose levels and type 2 diabetics may have improved blood sugar, lipids and insulin levels.. The average Glycemic Index of bananas is 52

\section{* Cancer fighter}

Yellow skin banana with dark spots on it is 8 times more effective in enhancing the property of white blood cells than green skin version. Recent researches show that fully ripe banana with the dark spots produce a compound called TNF (Tumor Necrosis Factor) This compound is a cytokine which is believed to have the potential to increase white blood cell count, thus enhancing the immunity and combating cancerous cell changes. Research on 61,000 women found that, of all the fruits, bananas gave the greatest protection against renal cancer. 
Women eating 5 bananas a week nearly halved their risk of renal cancer.

\section{* Prevents heart diseases}

It is also a very good source of vitaminB6 (pyridoxine), provides about 28\% of daily-recommended allowance. Pyridoxine is an important B-complex vitamin that has a beneficial role for the treatment of neuritis, and anemia. Further, it helps decrease homocystine (one of the causative factors in coronary artery disease (CHD) and stroke episodes) levels within the body. A study published in the Archives of Internal Medicine also confirms that eating high fiber foods, such as bananas, helps prevent heart disease.

\section{* Reduces depression}

Bananas contain a natural mood enhancer, tryptophan, a type of protein that the body converts to serotonin. Serotonin is known to make the body feel relax and improves good mood thus preventing seasonal affective disorder or depression. It also helps regulate good sleep patterns.

\section{* Strengthens bones}

Bananas contain probiotic bacteria, which helps the absorption of calcium in the body, thus strengthening the bones. Green bananas, also known as plantains, in addition to fructooligosaccharides contain short chain fatty acids (SCFA's) which is effective in strengthening the bones. The bottom line bananas, especially the green ones, are excellent for the bones. Also, the potassium in bananas helps to prevent calcium loss from the body. This lowers the risk of developing osteoporosis later in life.

\section{* Protects against ulcers}

Eating bananas regularly protects against stomach ulcers. Compounds in bananas create a thicker protective barrier in the stomach against hydrochloric acid. Bananas also contain protease inhibitors that work to eliminate certain bacteria in the stomach implicated as a major cause of stomach ulcers.

\section{* Improves digestion}

Banana contains fructo oligosaccharide, which helps to stimulate growth of good bacteria in the stomach, particularly the colon. It also produces enzymes that helps to improve the overall digestion. One banana has about $3 \mathrm{~g}$ of fiber. Dietary fiber keep our digestive processes running smoothly.

\section{* Natural moisturizer}

Banana contains Vitamin B6, C and a high percentage of water, which nourishes and hydrates the skin. These nutrients also help to maintain the elasticity of our skin. For instant solution to moisturise your dry skin, just apply pulp of a ripe banana on our face. Leave it on for 30 minutes before rinsing it off with lukewarm water. This application gives a pinkish glow to the skin.

\section{* Anti-ageing}

Bananas contains health promoting flavonoid poly-phenolic antioxidants such as lutein, zeaxanthin, $\beta$ and $\alpha$-carotenes in small amounts. These compounds help act as protective scavengers against oxygen-derived free radicals and reactive oxygen species (ROS) that play a role in aging and various disease processes.

Apply fresh peel on the face for 15 minutes every day to get the maximum benefit out of it. We also make a banana face pack by mashing half a banana and adding a teaspoon of rose water to it. Keep it on the face for about 30 minutes. After that, wash it off with water.

\section{* Treats acne and pimples:}

Bananas are very effective in treating acne and pimples. Take a small piece of the peel and rub it gently on the affected area. Do 
this till the peel turns brown. Then clean the

area with some water. Follow this simple routine thrice a day to get rid of those pimples.

\section{* Prevents insomnia:}

Bananas are rich in amino acids known as Tryptophan. This substance triggers production of melatonin (sleep hormone) which induces sleep. Eating banana a few hours before bedtime ensures a peaceful night's sleep.

\section{* Keeps hair healthy:}

Bananas are rich source of natural oils, carbohydrates, potassium and vitamins. These nutrients keep your hair healthy and smooth. They also nourish them, and prevent breakage and split ends. Mash a banana and add a little almond oil to it. Apply it on the hair, leave it on for about 15 minutes and then wash it off. Your hair will be revitalised and moisturised by the vitamin $\mathrm{C}$ and $\mathrm{A}$ present in bananas

\section{* . Treats cracked heels:}

Due to their moisturizing properties, bananas are great for our cracked heels as well. Just mash two bananas into a pulp, apply it on the feet for about 10 minutes before rinsing it off with water. The feet will become soft and smooth.

\section{* Get rid of puffy eyes}

Bananas are a rich source of potassium (400 $\mathrm{mg}$ potassium in a single medium-size banana)which can help to reduce the puffiness of our eyes instantly. Mash half a banana and apply it on the eyes for about 15 minutes. If we find this to be too messy, then just place banana peels on our swollen eyes.

\section{* Reduces inflammation due to}

\section{mosquito bites:}

Itching and swelling caused by mosquito bites can be reduced by rubbing the inside of a banana on it.

\section{Reference:}

- www.medindia.net-medicinal properties of bananas

- www.undergroundhealth.com-why bananas are great for immunity

- www.phytojournal.com-health benefits of banana

- saveourbones.com-boost calcium absorption with bananas

- www.medicalnewstoday.com-what are the health benefits of bananas

- www.medindia.net-home remedies and beauty 